\title{
General Election Hatena: The First Political Prediction Market in Japan
}

\author{
Hiroshi YAMAGUCHI ${ }^{1}$ \\ ${ }^{1}$ Faculty of Global Media Studies, Komazawa University.
}

\begin{abstract}
Last year, a Tokyo-based internet venture firm, Hatena Co., ran a political prediction market that was designed to predict the result of the General Election of the Lower House of the Diet, held on September 11, 2005. The market showed reasonable predictive ability that was comparable with the predictions of major newspapers and experts. In this paper, we summarize what happened at the first political prediction market in Japan, General Election Hatena, and discuss the future potential of prediction markets in Japan.
\end{abstract}

Keywords: prediction market, election, market design.

\section{Introduction}

The General Election of the Lower House held in September 11, 2005 was remarkable in which opinion of general public, especially among internet users, had significant influence. In this section we briefly review the background information on elections in Japan.

\subsection{The 9-11 General Election}

The Prime Minister Koizumi declared dissolution of the Lower House on August 10, 2005, which was the start of the so-called 9-11 General Election. His cabinet tried to privatize the Postal Office, but the Lower House rejected the bill. Those who opposed included the Diet members of Koizumi's own party, the Liberal Democratic Party (LDP).

Koizumi forced the opposing LDP members out of the party, and delegated "assassin" candidates to compete against them in the election. The bold move attracted broad attention from public, who had been longing for destroying "privileges" of bureaucrats at the Postal Office. Accelerated by media coverage, the supporting movement toward Koizumi overwhelmed that for other parties, resulted in the historic "landslide" victory of the LDP on the election.

\subsection{Role of internet opinions}

What was remarkable about the 9-11 General Election was that, for the first time in Japan, a mass of personal opinions flowing in internet had significant influence in formulating public opinions. As in other countries, in Japan the number of bloggers had reached a "critical math" so that discussions in the internet, including the prediction markets, became one of driving forces of the public opinion.

The construction of this paper is as follows. In Section 2, we briefly review prediction markets and discuss its meaning in the Japanese context. In Section 3 , we describe the company Hatena and its business. In Section 4, we see what happened in the prediction market and the result. In Section 5, we analyze the result. In Section 6, we draw implications from the experience and make some proposals for the future of political prediction markets in Japan.

\section{Political Prediction Markets}

Prediction markets have been recognized as an alternative approach to extract information from public. Researchers have so far shown that the market mechanism brings amazingly accurate results in predicting election results.

\subsection{Predictive power of prediction markets}

It is well known that security markets aggregate views on the future from investors, and extract an agreeable price based on the "wisdom of crowds." Prediction markets use this mechanism to make predictions. The first prediction market was developed by the University of Iowa in 1980s. The Iowa Electronic Markets (IEM), dealing mainly with political events such as US presidential elections, have so far proved that the overall prediction results were no worse than those of opinion polls (see [1]).

Now increasing number of companies run prediction markets. Companies including Hewlett Packard, Microsoft, and Siemens Austria launched experimental prediction markets to predict their own sales figures or project completion schedules. Trade Exchange Network in Ireland runs prediction markets 
TradeSports (sports-related) and InTrade (non sportsrelated) for betting. NewsFutures provide its prediction market engine to other organizations including the World Economic Forum.

IEM, and many experimental prediction markets run by firms use small amount of real money. And betting markets such as TradeSports also use real money. But the rest, such as that by NewsFutures, use imaginary money that has no direct connection to real value. [2] compared the predictive power of real and virtual money prediction markets, and concluded that there was no difference between the two.

One characteristic of prediction markets is that its predictive accuracy does not rely on the unbiasedness of the respondents. In case of IEM, majority of participants are Caucasian males, with relatively higher income, relative to average voters.

\subsection{Political prediction markets in Japanese context}

Prediction markets in the US showed effectiveness overall. However, introducing political prediction markets in Japan seemed to involve some difficulty. That is, ordinary Japanese people have not so accustomed to security markets as such compared to the people in the US. Japanese, unlike Americans, allocate only a small fraction of their savings to securities. If traders are not accustomed to how to behave in security markets, it is hard to expect that market mechanism functions.

\section{Hatena}

Hatena Co. is an internet venture company, established in 2001 by Junya Kondo. Hatena offers various internet services including a knowledge-sharing community site, blog hosting (first in Japan), social bookmarks, RSS readers, and so forth, on ASP basis. In all services, the firm exploits contribution of its users to provide the shared benefit among all users.

The system of the General Election Hatena was inherited from another prediction market run by the firm, called Hatena Idea. This is similar to the "idea futures" as in [3], but here a company, rather than the government, asks people about what to do.

Hatena Idea is a prediction market to predict "which improvement idea is to be implemented by Hatena?" Hatena regularly accepts all kinds of ideas to improve its service to customers, and these ideas were listed in its website. But it was difficult to determine the priority among the ideas. Once they tried to employ user votes, in which ideas that gained certain number of proponents were taken by the company. But this system had a fundamental problem; it was a zero-sum game between users and Hatena. Without paying any cost of improvements, users tended to ask for improvement ideas that were too costly to implement. Due to its limited resource, Hatena could not afford to implement all these improvement ideas.

Using the idea of prediction market, the firm tried to change the zero-sum game to a positive-sum one. By predicting the idea to be implemented, instead of asking the firm to implement an idea based on the personal wish, users put themselves in Hatena's place. They were discouraged to raise costly ideas, and tried to predict what idea was to be supported by Hatena as well as other users. Utilizing user community in this way fits well to the business model of Hatena.

\section{Experience}

In this section, we review the experience of the General Election Hatena. The authors obtained user demographics and transaction data from Hatena, and conducted a web-based survey for users in corporation with Hatena. 293 users responded to the survey.

\subsection{Participants}

The demographics of the participants were similar to the user base of Hatena, but it was clearly different from the actual voters.

The total number of participants was 1,149, and 90.8\% was male. According to Mr. Kondo, the president of Hatena, about $30 \%$ of Hatena users are females. In this sense, the participants of the General Election Hatena were skewed to males. Figure 1 compares the age structures of the survey, participant demographics, voters, and the Japanese population. Since no statistics on age structure of the voters of 911 election, we used that of the previous general election. Age structure of the population was taken from the result of census conducted in 2000.

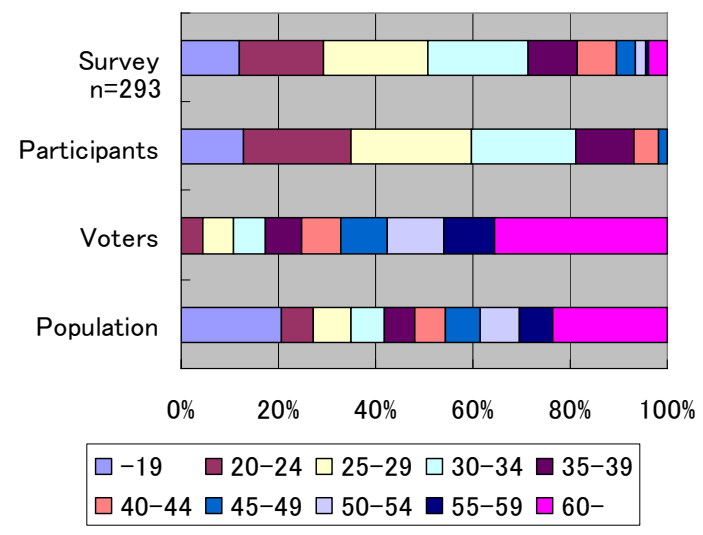

Figure 1: Comparison of Age Structure 


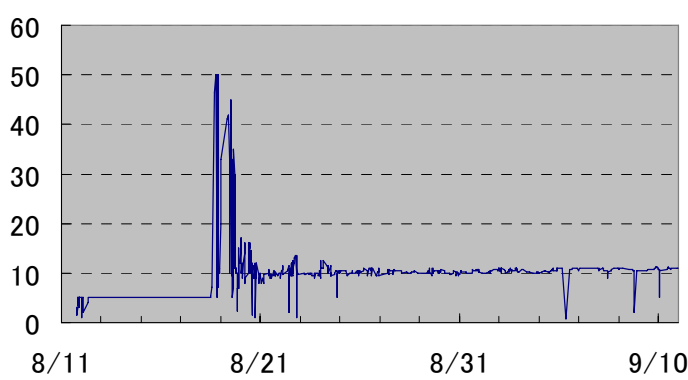

Figure 2: LDP: Price History (Aug. 11-Sept. 10)

As we see, the age structure of the survey (first bar) resembles to those of actual participants (second bar), meaning that the respondents of the survey was largely representative. But the age structure of the participants was greatly different from that of the voters (third bar).

\subsection{Market design}

General Election Hatena inherited the system from Hatena Idea, and incorporated its basic market design. Initially, Hatena issued 10,000 units of security for each of 8 political parties (Two new parties were added afterwards) on August 11. It used the same virtual money as in Hatena Idea, called "idea point." Issuing price was 1 point, but the upper limit price was set at 5 points. The market was basically pari-mutuel style, meaning that points invested by participants were allocated back to them according to the seat share. In addition, Hatena offered a prize of 100,000 points to motivate participants, which was also to be allocated according to the seat share.

Figure 2 shows the price history of the "LDP." The participants seemed to misunderstand the market design. When the first week after launch ended, the market capitalization of the all securities was more than 280,000 points, exceeding theoretical upper limit of 180,000 points $(10,000 p t$ times 8 securities plus 100,000 pt). This was clearly a "bubble," and since the upper limit price was set at 5 points, most securities reached at the limit.

A set of reform was implemented after August 18. Relaxing upper limit first drove the price of LDP up to unrealistically high level, but the misunderstanding among participants disappeared within a few days. In addition, Hatena issued security bundles to increase market liquidity, and displayed share of the market capitalization in addition to the price. Then by August 21 , the price converged to the reasonable level so that the prediction based on the market became useful. Figure 3 shows the market capitalization of LDP as the share of the total market capitalization and its moving average.

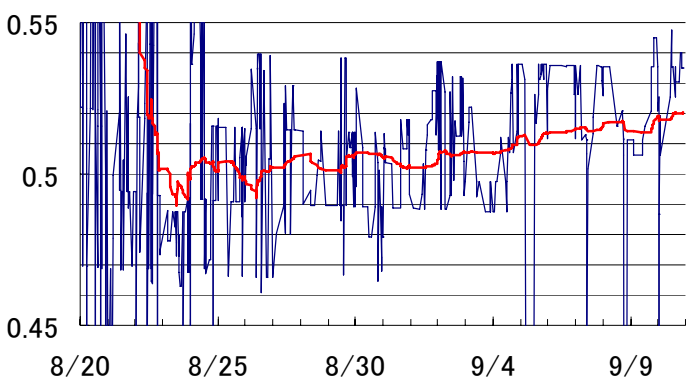

Figure 3: LDP Share of Market Capitalization (Aug. 20-Sept.10)

There still was a problem. In this design, participants could not predict the exact seat share because the expected value of reward points from Hatena per share changed as new security bundles were issued. Participants made predictions on a joint problem of "How many sets of security bundles are to be sold until the closing of the market?" and "What is the seat share of the respective parties?" Although that the overall effect seemed to be subtle, the design made prediction of participants more difficult.

\subsection{History and Prices}

The movement of the market capitalization after the initial turmoil as described above showed a moderate upward trend. This was somewhat consistent with the general trend of public opinion. Several downward spikes occurred, but quickly absorbed.

The moving average in Figure 3 clearly indicates that there are several “jumps" in LDP's predicted seat share. Namely, jumps occurred on August 28 (Sun), 30 (Tue), September 2 (Fri), 5 (Mon), and 8 (Thu). Note that these jumps took place in early morning.

Total number of transaction was 8,460, and 1,083 trades took place for LDP. When the market was closed on 24:00 of Sept. 10, the market capitalization of LDP reached $50.9 \%$ of the total market capitalization. Predictions and election results of parties are shown in Table 1 . The prediction showed a reasonable accuracy for small parties like JCP and SDP, but large discrepancy existed for LDP and DPJ.

\begin{tabular}{|l|r|r|}
\hline \multicolumn{1}{|c|}{ Party } & \multicolumn{1}{c|}{ Hatena } & \multicolumn{1}{c|}{ Result } \\
\hline LDP & $50.90 \%$ & $61.70 \%$ \\
\hline New Komeito & $8.40 \%$ & $6.50 \%$ \\
\hline DPJ & $31.30 \%$ & $23.50 \%$ \\
\hline JCP & $2.50 \%$ & $1.90 \%$ \\
\hline SDP & $1.30 \%$ & $1.50 \%$ \\
\hline Others & $5.60 \%$ & $5.00 \%$ \\
\hline
\end{tabular}

Table 1: Prediction and Results 


\begin{tabular}{|l|c|c|}
\hline & Seat Share & Date \\
\hline Result & $61.7 \%$ & 11-Sep \\
\hline Hatena & $50.9 \%$ & 10-Sep \\
\hline Predictions & & \\
\hline Nikkei & $56.3 \%$ & 5-Sep \\
\hline Asahi & $53.1 \%$ & 4-Sep \\
\hline Mainichi & $56.5 \%$ & 4-Sep \\
\hline Gendai & $50.8 \%$ & 3-Sep \\
\hline Mr. Kobayashi & $51.7 \%$ & 9-Sep \\
\hline Mr. Fukuoka & $51.7 \%$ & 9-Sep \\
\hline Mr. Miyagawa & $51.7 \%$ & 6-Sep \\
\hline Mr. Miura & $50.2 \%$ & 7-Sep \\
\hline
\end{tabular}

Table 2: Comparison of Predictions.

\section{Comparison of Prediction Results}

In this section we compare the prediction result of the General Election Hatena with those of other organizations and experts, by taking LDP as an example.

Table 2 shows the comparison of predictions of Hatena and other organizations with respect to the LDP's seat share. The organizations are newspaper companies (Nikkei, Asahi, and Mainichi) and a magazine publisher (Weekly Gendai). These predictions were prepared by these firms based on opinion polls that they (or their affiliated companies) conducted. And experts were well-known political commentators who expressed their predictions on major magazines.

As shown in Table 2, the vote share predicted by the General Election Hatena was less than the predictions of news paper companies, but close to those of experts. But all these predictions showed significantly lower figure compared to the actual election result. In fact as for the 9-11 General Election, no one could have predicted such a "landslide" victory of the LDP. This may be at least partially due to the fact that people could not make effective prediction under the newly introduced the single-member constituency system. In this sense, we conclude that the General Election Hatena showed reasonable predictive ability, no less accurate than those of other organizations based on opinion polls, and expert opinions.

\section{Implication and Proposal}

In this section, we conclude the discussion on the effectiveness of prediction markets in Japan, and propose some legislative measure to be taken so that the future of political prediction markets in Japan becomes more promising.

\subsection{How we evaluate the result?}

As in the previous section, the result of the General Election Hatena showed reasonable predictive ability compared to those of other predictions. It is noteworthy that the demographics of the participants were greatly different from that of the voters. The characteristic of prediction markets, irrelevance of "sample" bias, was confirmed here, too.

Moreover, it is noteworthy that Japanese people, who are less accustomed to security trading compared to those in the US, could behaved well during the trades at the General Election Hatena. Survey results showed relatively few participants had experienced security trading before. As online security trading becomes increasingly popular, people would become more accustomed to the user interface of prediction markets. This is a promising result when we think of the future potential of prediction markets in Japan.

\subsection{Need for legislation}

Based on the results above, there seems to be a potential for prediction markets to be utilized to political prediction as a complement to the existing methods such as opinion polls.

To pursue the direction further, however, it would be better for us to formally recognize the method as legitimate. The current General Election Act prohibits opinion polls during election campaign periods. This article aims to avoid biased polls that distort public opinion. So some people are afraid that political prediction markets potentially violate the article. But theoretically speaking, and as we saw above, prediction markets are not affected by bias of the "sample" (traders). But for clarification, it should be better to clearly state that prediction markets are not included in the "opinion polls" in the General Election Act.

\section{References}

[1] J. E. Berg, "Results from a Dozen Years of Election Futures Markets Research,” Working Paper, College of Business Administration, University of Iowa, 2000.

[2] E. Servan-Schreiber, J. Wolfers, D. M. Pennock, and B. Galebach, "Prediction Markets: Does Money Matter?” Electronic Markets 14, 3, pp. 243-251, 2004.

[3] R. Hanson, “Idea Futures,” Wired September 1995, pp. 125. 